\title{
On the Involute-Evolute of the Pseudonull Curve in Minkowski 3-Space
}

\author{
Ufuk Ozturk, ${ }^{1,2}$ Esra Betul Koc Ozturk, ${ }^{1,2}$ and Kazim Ilarslan ${ }^{3}$ \\ ${ }^{1}$ Department of Mathematics, Faculty of Sciences, University of Çankiri Karatekin, 18100 Çankiri, Turkey \\ ${ }^{2}$ School of Mathematics \& Statistical Sciences, Arizona State University, Room PSA442, Tempe, AZ 85287-1804, USA \\ ${ }^{3}$ Department of Mathematics, Faculty of Sciences and Arts, University of Kirikkale, 71450 Kirikkale, Turkey
}

Correspondence should be addressed to Esra Betul Koc Ozturk; ekocoztu@asu.edu

Received 6 June 2013; Accepted 10 September 2013

Academic Editor: Baolin Wang

Copyright (C) 2013 Ufuk Ozturk et al. This is an open access article distributed under the Creative Commons Attribution License, which permits unrestricted use, distribution, and reproduction in any medium, provided the original work is properly cited.

We have generalized the involute and evolute curves of the pseudonull curves $\alpha$ in $\mathbb{E}_{1}^{3}$; that is, $\alpha$ is a spacelike curve with a null principal normal. Firstly, we have shown that there is no involute of the pseudonull curves $\alpha$ in $\mathbb{E}_{1}^{3}$. Secondly, we have found relationships between the evolute curve $\beta$ and the pseudonull curve $\alpha$ in $\mathbb{E}_{1}^{3}$. Finally, some examples concerning these relations are given.

\section{Introduction}

The general theory of curves in an Euclidean space (or more generally in a Riemannian manifold) was developed a long time ago, so now, we have a deep knowledge of its local geometry as well as its global geometry. In the theory of curves in Euclidean space, one of the important and interesting problems is the characterizations of a regular curve. And, in particular, the involute-evolute of a given curve is a well-known concept in the classical differential geometry (see [1]).

At the beginning of the twentieth century, A. Einstein's theory opened a door for use of new geometries. One of them is simultaneously the geometry of special relativity and the geometry induced on each fixed tangent space of an arbitrary Lorentzian manifold and was introduced and some of classical differential geometry topics have been treated by the researchers. According to reference (see $[2,3]$ ), the involute and evolute curves of the spacelike curve $\alpha$ in $\mathbb{E}_{1}^{3}$ with a spacelike binormal or a spacelike principal normal in Minkowski 3-space have been investigated. Also, Bükcü and Karacan studied the involute and evolute curves of the timelike curve in Minkowski 3-space [4].

In this paper, we have generalized the involute and evolute curves of the pseudonull curves $\alpha$ in $\mathbb{E}_{1}^{3}$; that is, $\alpha$ is a spacelike curve with a null principal normal. Firstly, we have shown that there is no involute of the pseudonull curves $\alpha$ in $\mathbb{E}_{1}^{3}$. Secondly, we have found relationships between the evolute curve $\beta$ and the pseudonull curve $\alpha$ in $\mathbb{E}_{1}^{3}$. Finally, some examples concerning these relations are given.

\section{Preliminaries}

The Minkowski 3-space $\mathbb{E}_{1}^{3}$ is the Euclidean 3-space $\mathbb{E}^{3}$ provided with the standard flat metric given by

$$
g=-d x_{1}^{2}+d x_{2}^{2}+d x_{3}^{2}
$$

where $\left(x_{1}, x_{2}, x_{3}\right)$ is a rectangular coordinate system of $\mathbb{E}_{1}^{3}$. Since $g$ is an indefinite metric, recall that a vector $v \in \mathbb{E}_{1}^{3}$ can have one of three Lorentzian causal characters: it can be spacelike if $g(v, v)>0$ or $v=0$, timelike if $g(v, v)<0$, and null (lightlike) if $g(v, v)=0$ and $v \neq 0$. In particular, the norm (length) of a nonnull vector $v$ is given $\|v\|=$ $\sqrt{|g(v, v)|}$, and two vectors $v$ and $w$ are said to be orthogonal, if $g(v, w)=0$. A lightlike vector $X$ is said to be positive (resp., negative) if and only if $x_{1}>0$ (resp., $x_{1}<0$ ) and a timelike vector $X$ is said to be positive (resp., negative) if and only if $x_{1}>0$ (resp., $\left.x_{1}<0\right)$. 
Next, recall that an arbitrary curve $\alpha=\alpha(s)$ in $\mathbb{E}_{1}^{3}$ can locally be spacelike, timelike, or null (lightlike), if all of its velocity vectors $\alpha^{\prime}(s)$ are, respectively, spacelike, timelike, or null (lightlike). If $g\left(\alpha^{\prime}(s), \alpha^{\prime}(s)\right)= \pm 1$, the nonnull curve $\alpha$ is said to be of unit speed (or parameterized by arc length function $s$ ).

We denote by $\{\vec{T}, \vec{N}, \vec{B}\}$ the moving Frenet frame along the curve $\alpha(s)$. Then $\vec{T}, \vec{N}$, and $\vec{B}$ are the tangent, the principal normal, and the binormal vector of the curve $\alpha$, respectively. Depending on the causal character of the curve $\alpha$, we have the following Frenet-Serret formulas.

If $\alpha$ is a null space curve with a spacelike principal normal $\vec{N}$, then the following Frenet formulas hold

$$
\vec{T}^{\prime}=\kappa \vec{N}, \quad \vec{N}^{\prime}=\tau \vec{T}-\kappa \vec{B}, \quad \vec{B}^{\prime}=-\tau \vec{N},
$$

where

$$
\begin{array}{ll}
g(\vec{T}, \vec{T})=g(\vec{B}, \vec{B})=0, & g(\vec{N}, \vec{N})=1, \\
g(\vec{T}, \vec{N})=g(\vec{N}, \vec{B})=0, & g(\vec{T}, \vec{B})=1 .
\end{array}
$$

For a null curve, $\kappa$ can take only two values: $\kappa=0$ when $\alpha$ is a null straight line or $\kappa=1$ in all other cases.

If $\alpha$ is a pseudonull curve, that is, $\alpha$ is a spacelike curve with a null principal normal $N$, then the following Frenet formulas hold:

$$
\vec{T}^{\prime}=\kappa \vec{N}, \quad \vec{N}^{\prime}=\tau \vec{N}, \quad \vec{B}^{\prime}=-\kappa \vec{T}-\tau \vec{B},
$$

where

$$
\begin{array}{ll}
g(\vec{T}, \vec{T})=1, & g(\vec{N}, \vec{N})=g(\vec{B}, \vec{B})=0, \\
g(\vec{N}, \vec{B})=1, & g(\vec{T}, \vec{N})=g(\vec{T}, \vec{B})=0 .
\end{array}
$$

For a pseudonull curve, $\kappa$ can take only two values: $\kappa=0$ when $\alpha$ is a straight line or $\kappa=1$ in all other cases.

If $\alpha$ is a spacelike space curve with a spacelike principal normal $\vec{N}$, then the following Frenet formulas hold

$$
\vec{T}^{\prime}=\kappa \vec{N}, \quad \vec{N}^{\prime}=-\kappa \vec{T}+\tau \vec{B}, \quad \vec{B}^{\prime}=\tau \vec{N},
$$

where

$$
\begin{gathered}
g(\vec{T}, \vec{T})=g(\vec{N}, \vec{N})=1, \quad g(\vec{B}, \vec{B})=-1, \\
g(\vec{T}, N)=g(\vec{N}, B)=g(\vec{T}, \vec{B})=0 .
\end{gathered}
$$

If $\alpha$ is a spacelike space curve with a timelike principal normal $\vec{N}$, then the following Frenet formulas hold

$$
\vec{T}^{\prime}=\kappa \vec{N}, \quad \vec{N}^{\prime}=\kappa \vec{T}+\tau \vec{B}, \quad \vec{B}^{\prime}=\tau \vec{N},
$$

where

$$
\begin{gathered}
g(\vec{T}, \vec{T})=g(\vec{B}, \vec{B})=1, \quad g(\vec{N}, \vec{N})=-1, \\
g(\vec{T}, \vec{N})=g(\vec{N}, \vec{B})=g(\vec{T}, \vec{B})=0 .
\end{gathered}
$$

If $\alpha$ is a timelike space curve, then the following Frenet formulas hold

$$
\vec{T}^{\prime}=\kappa \vec{N}, \quad \vec{N}^{\prime}=\kappa \vec{T}+\tau \vec{B}, \quad \vec{B}^{\prime}=-\tau \vec{N},
$$

where

$$
\begin{gathered}
g(\vec{T}, \vec{T})=-1, \quad g(\vec{N}, \vec{N})=g(\vec{B}, \vec{B})=1, \\
g(\vec{N}, \vec{B})=g(\vec{T}, \vec{N})=g(\vec{T}, \vec{B})=0 ;
\end{gathered}
$$

see [5].

If the curve $\alpha(t)$ is nonunit speed, then

$$
\begin{aligned}
& \kappa(s)=\frac{\left\|\alpha^{\prime}(t) \wedge \alpha^{\prime \prime}(t)\right\|}{\left\|\alpha^{\prime}(t)\right\|^{3}}, \\
& \tau(s)=\frac{\operatorname{det}\left(\alpha^{\prime}(t), \alpha^{\prime \prime}(t), \alpha^{\prime \prime \prime}(t)\right)}{\left\|\alpha^{\prime}(t) \wedge \alpha^{\prime \prime}(t)\right\|^{2}} .
\end{aligned}
$$

If the curve $\alpha(s)$ is unit speed, then

$$
\begin{aligned}
& \kappa(s)=\left\|\alpha^{\prime \prime}(s)\right\|, \\
& \tau(s)=\left\|\vec{B}^{\prime}(s)\right\| ;
\end{aligned}
$$

see [6].

In this study we are going to have the $\alpha$ curve as nonline pseudonull curve or $\kappa=1$.

\section{The Involute of the Pseudonull Curve}

Definition 1. Let a pseudonull curve $\alpha: I \rightarrow \mathbb{E}_{1}^{3}$ and a curve $\beta: I \rightarrow \mathbb{E}_{1}^{3}$ be given. For all $s \in I$, then the curve $\beta$ is called the involute of the curve $\alpha$, if the tangent vector of the curve $\alpha$ at the point $\alpha(s)$ passes through the tangent at the point $\beta(s)$ of the curve $\beta$ and

$$
g\left(\vec{T}(s), \vec{T}^{*}(s)\right)=0,
$$

where $\{\vec{T}, \vec{N}, \vec{B}\}$ and $\left\{\vec{T}^{*}, \vec{N}^{*}, \vec{B}^{*}\right\}$ are Frenet frames of $\alpha$ and $\beta$, respectively.

This definition suffices to define this curve mate as

$$
\beta(s)=\alpha(s)+\lambda(s) \vec{T}(s)
$$

(see Figure 1).

Theorem 2. Let $\alpha: I \rightarrow \mathbb{E}_{1}^{3}$ be a pseudonull space curve with curvature functions $\kappa(s)=1$ and $\tau(s) \neq 0$. Then, there is no involute of the curve $\alpha$ in Minkowski 3-space $\mathbb{E}_{1}^{3}$.

Proof. Let $\beta: I \rightarrow \mathbb{E}_{1}^{3}$ be involute of $\alpha$ in $\mathbb{E}_{1}^{3}$. We assume that $\beta$ is distinct from $\alpha$. Then we can write

$$
\beta(s)=\alpha(s)+\lambda(s) \vec{T}(s),
$$




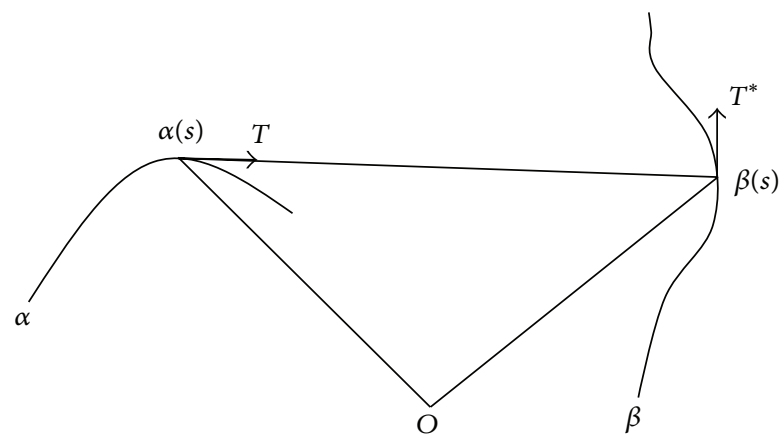

FIgURE 1: Involute curves.

where $\lambda$ is a $C^{\infty}$-function on $I$. Differentiating (17) and by using Frenet formulas given in (4), we get

$$
\vec{T}^{*}(s)=\left(1+\frac{d \lambda}{d s}\right) \vec{T}(s)+\lambda(s) \vec{N}(s) .
$$

If we take the inner product with $\vec{T}(s)$ on both sides of (18), we have

$$
\begin{aligned}
g\left(\vec{T}(s), \vec{T}^{*}(s)\right)= & \left(1+\frac{d \lambda}{d s}\right) g(\vec{T}(s), \vec{T}(s)) \\
& +\lambda(s) g(\vec{N}(s), \vec{T}(s)) .
\end{aligned}
$$

Recalling definition of the involute curve couple, $g(\vec{T}(s)$, $\left.\vec{T}^{*}(s)\right)=0$ and, by using (5), we get

$$
1+\frac{d \lambda}{d s}=0
$$

for all $s \in I$. Thus, since $c \in \mathbb{R}-\{0\}$, we get $\lambda(s)=c-s$; that is, $\lambda(s)$ is a nonzero constant. In this case, we can write (17) as follows:

$$
\beta(s)=\alpha(s)+(c-s) \vec{T}(s),
$$

and from (18) we get

$$
\vec{T}^{*}(s)=\lambda(s) \vec{N}(s) .
$$

Here we notice that

$$
g\left(\vec{T}^{*}(s), \vec{T}^{*}(s)\right)=\lambda^{2}(s) g(\vec{N}(s), \vec{N}(s)),
$$

and by using (5) we get

$$
g\left(\vec{T}^{*}(s), \vec{T}^{*}(s)\right)=0 .
$$

Thus, since $g\left(\vec{T}^{*}(s), \vec{T}^{*}(s)\right)=0$, we get that $\vec{T}^{*}$ is a null vector; that is, curve $\beta$ is a null curve.

Differentiating (24) with respect to $s$ and by using Frenet formulas given in (4), we have

$$
\kappa^{*}(s) \vec{N}^{*}(s)=(\lambda(s) \tau(s)-1) \vec{N}(s),
$$

and by using (5) we get

$$
\left(\kappa^{*}(s)\right)^{2} g\left(\vec{N}^{*}(s), \vec{N}^{*}(s)\right)=0 .
$$

Since $\kappa^{*}(s) \neq 0$ for all $s \in I$, we get

$$
g\left(\vec{N}^{*}(s), \vec{N}^{*}(s)\right)=0 .
$$

Thus, we obtain that $\vec{N}^{*}$ is a null vector. This is a contradiction with null curve $\beta$. Then, there is no involute of the curve $\alpha$ in Minkowski 3-space $\mathbb{E}_{1}^{3}$.

\section{The Evolute of the Pseudonull Curve}

Definition 3. Let the pseudonull curve $\alpha$ and a curve $\beta$ with the same interval be given. For all $s \in I$, then the curve $\beta$ is called the evolute of the curve $\alpha$, if the tangent vector of the curve $\beta$ at the point $\beta(\mathrm{s})$ passes through the tangent at the point $\alpha(s)$ of the curve $\alpha$ and

$$
g\left(\vec{T}(s), \vec{T}^{*}(s)\right)=0,
$$

where $\{\vec{N}, \vec{N}, \vec{B}, \kappa=1, \tau\}$ and $\left\{\vec{N}^{*}, \vec{N}^{*}, \vec{B}^{*}, \kappa^{*}, \tau^{*}\right\}$ are Frenet frames and curvatures of $\alpha$ and $\beta$, respectively.

This definition suffices to define this curve mate as

$$
\beta(s)=\alpha(s)+\lambda(s) \vec{N}(s)+\mu(s) \vec{B}(s) .
$$

Theorem 4. Let a space curve $\beta$ be the evolute of the pseudonull curve $\alpha$; then for all $s \in I$

$$
\mu(s)=1,
$$

where $\{\vec{T}, \vec{N}, \vec{B}, \kappa=1, \tau\}$ and $\left\{\vec{T}^{*}, \vec{N}^{*}, \vec{B}^{*}, \kappa^{*}, \tau^{*}\right\}$ are Frenet frames and curvatures of $\alpha$ and $\beta$, respectively.

Proof. The tangent of the curve $\beta$ at the point $\beta(s)$ is the line constructed by the vector $\vec{T}^{*}(s)$. Then we can write

$$
\beta(s)=\alpha(s)+\lambda(s) \vec{N}(s)+\mu(s) \vec{B}(s),
$$

where $\lambda$ and $\mu$ are a $C^{\infty}$-function on $I$. Differentiating (31) and by using Frenet formulas given in (4), we get

$$
\begin{aligned}
\beta^{\prime}(s)= & (1-\mu(s)) \vec{T}(s)+\left(\lambda^{\prime}(s)+\lambda(s) \tau(s)\right) \vec{N}(s) \\
& +\left(\mu^{\prime}(s)-\mu(s) \tau(s)\right) \vec{B}(s)
\end{aligned}
$$

and, from $\beta^{\prime}(s)=\vec{T}^{*}(s)$,

$$
\begin{aligned}
\vec{T}^{*}(s)= & (1-\mu(s)) \vec{T}(s)+\left(\lambda^{\prime}(s)+\lambda(s) \tau(s)\right) \vec{N}(s) \\
& +\left(\mu^{\prime}(s)-\mu(s) \tau(s)\right) \vec{B}(s) .
\end{aligned}
$$

If we take the inner product with $\vec{T}(s)$ on both sides of (33), we have

$$
\begin{aligned}
g(\vec{T}(s) & \left., \vec{T}^{*}(s)\right) \\
= & (1-\mu(s)) g(\vec{T}(s), \vec{T}(s)) \\
& +\left(\lambda^{\prime}(s)+\lambda(s) \tau(s)\right) g(\vec{N}(s), \vec{T}(s)) \\
& +\left(\mu^{\prime}(s)-\mu(s) \tau(s)\right) g(\vec{B}(s), \vec{T}(s)) .
\end{aligned}
$$


Recalling definition of the evolute curve couple, $g(\vec{T}(s)$, $\left.\vec{T}^{*}(s)\right)=0$, and by using (5), we get

$$
\mu(s)=1,
$$

for all $s \in I$. In this case, we can write (31) as follows:

$$
\beta(s)=\alpha(s)+\lambda(s) \vec{N}(s)+\vec{B}(s),
$$

and from (35) we get

$$
\vec{T}^{*}(s)=\left(\lambda^{\prime}(s)+\lambda(s) \tau(s)\right) \vec{N}(s)-\tau(s) \vec{B}(s) .
$$

Let $\{\vec{T}, \vec{N}, \vec{B}, \kappa=1, \tau\}$ and $\left\{\vec{T}^{*}, \vec{N}^{*}, \vec{B}^{*}, \kappa^{*}, \tau^{*}\right\}$ be Frenet frames and curvatures of $\alpha$ and $\beta$, respectively. If the space curve $\beta$ is the evolute of the pseudonull curve $\alpha$, then, from Theorem 4, we can write

$$
\begin{gathered}
\beta(s)=\alpha(s)+\lambda(s) \vec{N}(s)+\vec{B}(s), \\
\beta^{\prime}(s)=\vec{T}^{*}(s)=\left(\lambda^{\prime}(s)+\lambda(s) \tau(s)\right) \vec{N}(s)-\tau(s) \vec{B}(s),
\end{gathered}
$$

where $\lambda$ is a $C^{\infty}$-function on $I$.

Since this line passes through the point $\alpha(s)$, the vector $\beta(s)-\alpha(s)$ is perpendicular to the vector $\vec{T}(s)$. From (36) and (37), the vector field $\beta^{\prime}(s)=\vec{T}^{*}(s)$ is parallel to the vector field $\beta(s)-\alpha(s)$. Then, we have

$$
\frac{\lambda^{\prime}(s)+\lambda(s) \tau(s)}{\lambda(s)}=\frac{-\tau(s)}{1} .
$$

4.1. Special Cases. If $\lambda(s)=0$ for all $s \in I$, then (39) is not defined. But, from (36) and (37), we get

$$
\begin{gathered}
\beta(s)=\alpha(s)+\vec{B}(s), \\
\beta^{\prime}(s)=\vec{T}^{*}(s)=-\tau(s) \vec{B}(s) .
\end{gathered}
$$

Here we notice that

$$
g\left(\vec{T}^{*}(s), \vec{T}^{*}(s)\right)=\tau^{2}(s) g(\vec{B}(s), \vec{B}(s))
$$

and by using (5) we get

$$
g\left(\vec{T}^{*}(s), \vec{T}^{*}(s)\right)=0 .
$$

Thus, since $g\left(\vec{T}^{*}(s), \vec{T}^{*}(s)\right)=0$, we get that $\vec{T}^{*}$ is a null vector; that is, the curve $\beta$ is a null curve. Differentiating (41) and by using Frenet formulas given in (4), we have

$$
\kappa^{*}(s) \vec{N}^{*}(s)=\tau(s) \vec{T}(s)+\left(\tau^{2}(s)-\tau^{\prime}(s)\right) \vec{B}(s),
$$

and from (5) we get

$$
\left(\kappa^{*}(s)\right)^{2} g\left(\vec{N}^{*}(s), \vec{N}^{*}(s)\right)=\tau^{2}(s) .
$$

Since the space curve $\beta$ is a null curve, then from $g\left(\vec{N}^{*}(s), \vec{N}^{*}(s)\right)=1$ and $\kappa^{*}(s)=1$ we get

$$
\tau(s)=\mp 1 \text {. }
$$

Case 1. If $\tau(s)=-1$ from (46), then we can write (41) and (44) as follows:

$$
\begin{gathered}
\vec{T}^{*}(s)=\vec{B}(s), \\
\vec{N}^{*}(s)=-\vec{T}(s)+\vec{B}(s) .
\end{gathered}
$$

Differentiating (48) and by using Frenet formulas given in (2) and (4), we get

$$
\vec{B}^{*}(s)=\vec{T}(s)+\vec{N}(s)-\frac{1}{2} \vec{B}(s) .
$$

By using Frenet formulas given in (2), we get

$$
\tau^{*}(s)=\frac{1}{2} .
$$

Conclusion 1. Let a space curve $\beta$ be the evolute of the pseudo null curve $\alpha$ in $\mathbb{E}_{1}^{3}$ and let $\kappa^{*}$ and $\tau^{*}$ are the curvatures of the curve $\beta$. If the torsion $\tau$ of the curve $\alpha$ is

$$
\tau=-1 \text {, }
$$

for all $s \in I$, then $\alpha$ and $\beta$ are helices and the curvatures of the curve $\beta$ are

$$
\kappa^{*}(s)=1, \quad \tau^{*}(s)=\frac{1}{2},
$$

for all $s \in I$.

Case 2. If $\tau(s)=1$ from (46), then we can write (41) and (44) as follows:

$$
\begin{gathered}
\vec{T}^{*}(s)=-\vec{B}(s), \\
\vec{N}^{*}(s)=\vec{T}(s)+\vec{B}(s) .
\end{gathered}
$$

Differentiating (54) and by using Frenet formulas given in (2) and (4), we get

$$
\vec{B}^{*}(s)=\vec{T}(s)-\vec{N}(s)-\frac{1}{2} \vec{B}(s) .
$$

By using Frenet formulas given in (2), we get

$$
\tau^{*}(s)=\frac{1}{2} .
$$

Conclusion 2. Let a space curve $\beta$ be the evolute of the pseudo null curve $\alpha$ in $\mathbb{E}_{1}^{3}$ and let $\kappa^{*}$ and $\tau^{*}$ are the curvatures of the curve $\beta$. If the torsion $\tau$ of the curve $\alpha$ is

$$
\tau=1,
$$

for all $s \in I$, then $\alpha$ and $\beta$ are helices and the curvatures of the curve $\beta$ are

$$
\kappa^{*}(s)=1, \quad \tau^{*}(s)=\frac{1}{2},
$$

for all $s \in I$.

$$
\begin{aligned}
& \text { If } \lambda(s)=c \neq 0(c=\text { constant }) \text {, then from (39) we get } \\
& \qquad \tau(s)=-\frac{\lambda^{\prime}(s)}{2 \lambda(s)}
\end{aligned}
$$

and if $\tau(s)=0$ for all $s$, then the curve $\beta$ is not defined. 
4.2. Special Cases. If $\lambda(s) \neq c$ ( $c=$ constant), then from (39) we get

$$
\tau(s)=-\frac{\lambda^{\prime}(s)}{2 \lambda(s)} .
$$

From (4), (5), and (37), we get

$$
g\left(\vec{T}^{*}(s), \vec{T}^{*}(s)\right)=-2 \tau(s)\left[\lambda^{\prime}(s)+\lambda(s) \tau(s)\right],
$$

and by using (60) we get

$$
g\left(\vec{T}^{*}(s), \vec{T}^{*}(s)\right)=\frac{\left[\lambda^{\prime}(s)\right]^{2}}{2 \lambda(s)} .
$$

Case 3. If $\beta$ is a unit spacelike curve, then from (62) we have

$$
\left[\lambda^{\prime}(s)\right]^{2}-2 \lambda(s)=0 .
$$

Solving the differential equation (63), we get

$$
\lambda(s)=\frac{s^{2}}{2}+c_{1} s+c_{2}, \quad \forall s \in I,
$$

where $c_{1}$ and $c_{2}$ are constant. Then, from (36) we can write

$$
\beta(s)=\alpha(s)+\left(\frac{s^{2}}{2}+c_{1} s+c_{2}\right) \vec{N}(s)+\vec{B}(s) .
$$

Case 4. If $\beta$ is a unit timelike curve, then from (62) we have

$$
\left[\lambda^{\prime}(s)\right]^{2}+2 \lambda(s)=0 .
$$

Solving the differential equation (65), we get

$$
\lambda(s)=-\frac{s^{2}}{2}+c_{1} s+c_{2}, \quad \forall s \in I,
$$

where $c_{1}$ and $c_{2}$ are constant. Then, from (36) we can write

$$
\beta(s)=\alpha(s)+\left(-\frac{s^{2}}{2}+c_{1} s+c_{2}\right) \vec{N}(s)+\vec{B}(s) .
$$

Case 5. If $\beta$ is a null curve, then from (62) we have

$$
\left[\lambda^{\prime}(s)\right]^{2}=0 .
$$

Solving of the differential equation (65), we get

$$
\lambda(s)=c, \quad \forall s \in I,
$$

where $c$ is constant. This is a contraction with $\lambda(s) \neq c(c=$ constant). Then, the curve $\beta$ is not defined.

\section{Example}

Let $\alpha(s)$ be a unit speed pseudonull curve in the Minkowski 3 -space $\mathbb{E}_{1}^{3}$ with parameter equation (see Figure 2)

$$
\alpha(s)=\left(s^{3}+s^{2}, s^{3}+s^{2}, s\right) .
$$

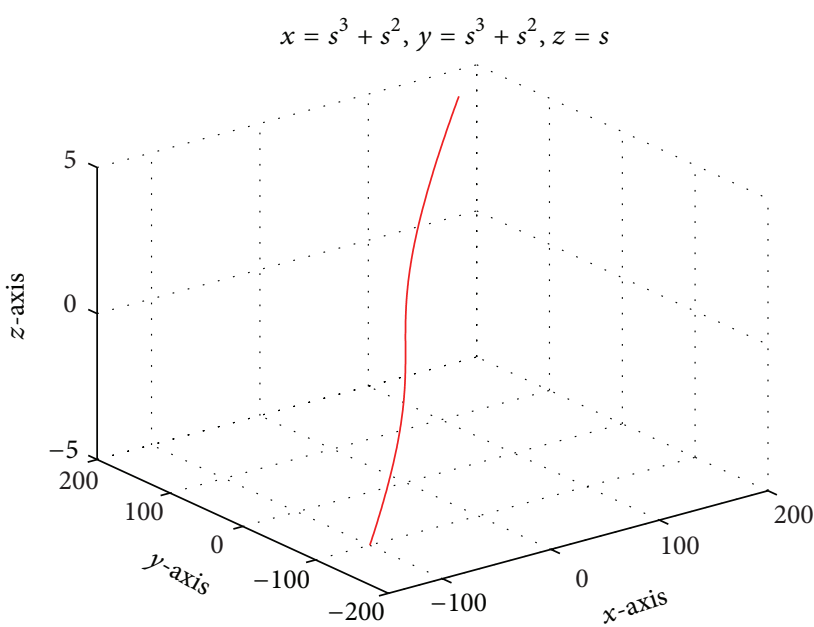

Figure 2: The pseudonull curve $\alpha(s)$ in $\mathbb{E}_{1}^{3}$.

$$
\begin{aligned}
& x=s^{3}+s^{2}-\left(\left(\left(3 s^{2}+2 s\right)^{2}+1\right) /(12 s+4)\right), \\
& y=s^{3}+s^{2}+\left(\left(1-\left(3 s^{2}+2 s\right)^{2}\right) /(12 s+4)\right), \\
& z=s-\left(\left(3 s^{2}+2 s\right) /(6 s+2)\right) .
\end{aligned}
$$

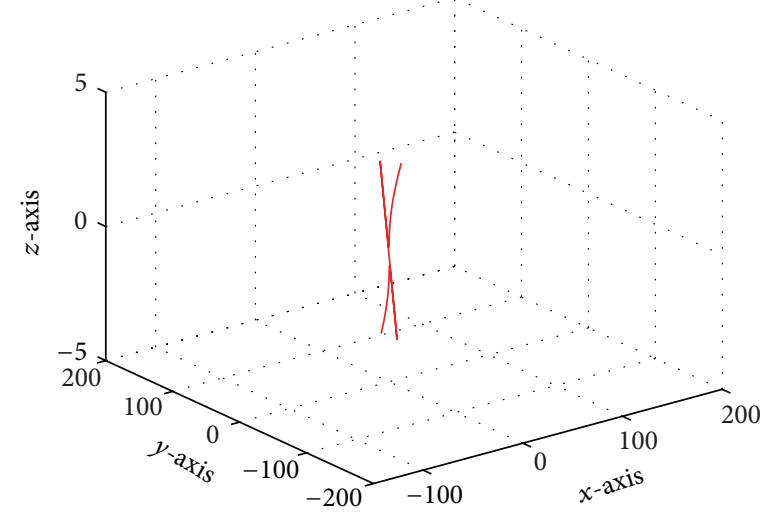

FIgURE 3: The evolute curve $\beta(s)$ of the pseudonull curve $\alpha(s)$ in $\mathbb{E}_{1}^{3}$.

$$
\begin{aligned}
& x=4 s^{3}+2 s^{2}-\left(\left(\left(3 s^{2}+2 s\right)^{2}+1\right) /(12 s+4)\right)+\left(6 s^{2}+2 s\right)+(6 s+2), \\
& y=4 s^{3}+2 s^{2}+\left(\left(1-\left(3 s^{2}+2 s\right)^{2}\right) /(12 s+4)\right)+\left(6 s^{2}+2 s\right)+(6 s+2), \\
& z=s-\left(\left(3 s^{2}+2 s\right) /(6 s+2)\right) .
\end{aligned}
$$

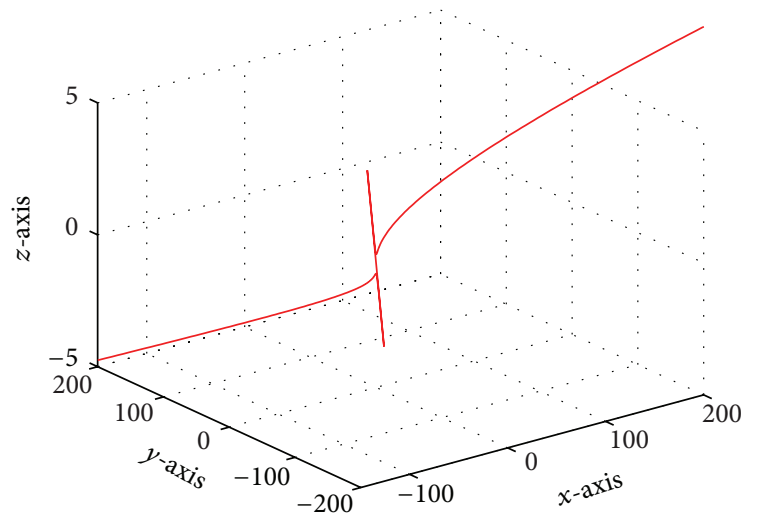

FIgURE 4: The evolute curve $\beta(s)$ of the pseudonull curve $\alpha(s)$ in $\mathbb{E}_{1}^{3}$. 


$$
\begin{aligned}
& x=-2 s^{3}-\left(\left(\left(3 s^{2}+2 s\right)^{2}+1\right) /(12 s+4)\right)+\left(6 s^{2}+2 s\right)+(6 s+2) \\
& y=-2 s^{3}+2 s^{2}+\left(\left(\left(1-\left(3 s^{2}+2 s\right)^{2}\right) /(12 s+4)\right)+\left(6 s^{2}+2 s\right)+(6 s+2)\right. \\
& z=s-\left(\left(3 s^{2}+2 s\right) /(6 s+2)\right)
\end{aligned}
$$

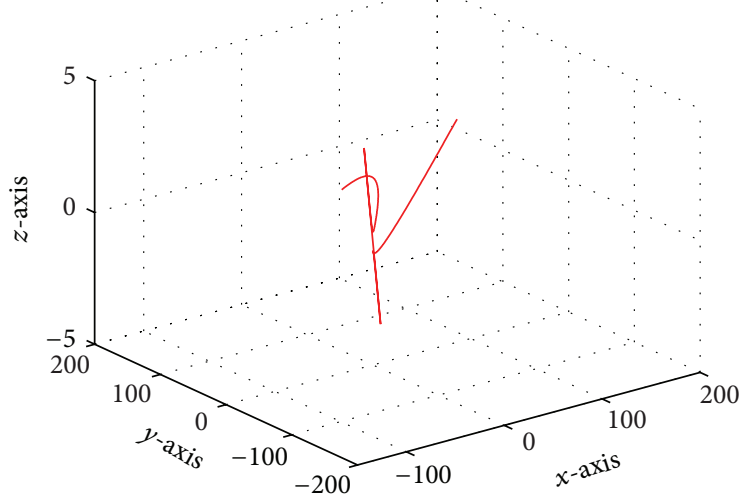

FIGURE 5: The evolute curve $\beta(s)$ of the pseudonull curve $\alpha(s)$ in $\mathbb{E}_{1}^{3}$.

Then we have the Frenet frame vectors as follows:

$$
\begin{gathered}
\vec{T}(s)=\alpha^{\prime}(s)=\left(3 s^{2}+2 s, 3 s^{2}+2 s, 1\right), \\
\vec{N}(s)=\alpha^{\prime \prime}(s)=(6 s+2,6 s+2,0), \\
\vec{B}(s)=\left(-\frac{\left(3 s^{2}+2 s\right)^{2}+1}{12 s+4}, \frac{1-\left(3 s^{2}+2 s\right)^{2}}{12 s+4},-\frac{3 s^{2}+2 s}{6 s+2}\right),
\end{gathered}
$$

where $\vec{T}(s)$ is spacelike vector and $\vec{N}(s)$ and $\vec{B}(s)$ are null vectors for all $s$. Moreover the curvatures $\kappa$ and $\tau$ of $\alpha$ are as follows:

$$
\kappa(s)=1, \quad \tau(s)=\frac{6}{6 s+1} .
$$

Let the space curve $\beta$ with the same interval be the evolute of the pseudonull curve $\alpha$. Then we can write

$\beta(s)$

$$
\begin{aligned}
= & \alpha(s)+\lambda(s) \vec{N}(s)+\mu(s) \vec{B}(s) \\
= & \left(3 s^{2}+2 s, 3 s^{2}+2 s, 1\right)+\lambda(s)(6 s+2,6 s+2,0) \\
& +\mu(s)\left(-\frac{\left(3 s^{2}+2 s\right)^{2}+1}{12 s+4}, \frac{1-\left(3 s^{2}+2 s\right)^{2}}{12 s+4},-\frac{3 s^{2}+2 s}{6 s+2}\right) .
\end{aligned}
$$

(1) From (40), the curve $\beta$ is given by (see Figure 3 )

$$
\begin{array}{r}
\beta(s)=\left(s^{3}+s^{2}-\frac{\left(3 s^{2}+2 s\right)^{2}+1}{12 s+4}, s^{3}+s^{2}\right. \\
\left.+\frac{1-\left(3 s^{2}+2 s\right)^{2}}{12 s+4}, s-\frac{3 s^{2}+2 s}{6 s+2}\right) .
\end{array}
$$

(2) From (65), the curve $\beta$ is given by (see Figure 4)

$\beta(s)$

$$
\begin{aligned}
& =\left(4 s^{3}+2 s^{2}-\frac{\left(3 s^{2}+2 s\right)^{2}+1}{12 s+4}\right. \\
& +c_{1}\left(6 s^{2}+2 s\right)+c_{2}(6 s+2), \\
& 4 s^{3}+2 s^{2}+\frac{1-\left(3 s^{2}+2 s\right)^{2}}{12 s+4} \\
& \left.+c_{1}\left(6 s^{2}+2 s\right)+c_{2}(6 s+2), s-\frac{3 s^{2}+2 s}{6 s+2}\right) \text {. }
\end{aligned}
$$

(3) From (68), the curve $\beta$ is given by (see Figure 5)

$\beta(s)$

$$
\begin{gathered}
=\left(-2 s^{3}-\frac{\left(3 s^{2}+2 s\right)^{2}+1}{12 s+4}+c_{1}\left(6 s^{2}+2 s\right)+c_{2}(6 s+2),\right. \\
-2 s^{3}+2 s^{2}+\frac{1-\left(3 s^{2}+2 s\right)^{2}}{12 s+4} \\
\left.+c_{1}\left(6 s^{2}+2 s\right)+c_{2}(6 s+2), s-\frac{3 s^{2}+2 s}{6 s+2}\right) .
\end{gathered}
$$

\section{Acknowledgment}

The authors would like to thank the referee for his/her helpful suggestions and comments which significantly improved the first version of the paper.

\section{References}

[1] R. S. Millman and G. D. Parker, Elements of Differential Geometry, Prentice-Hall, Englewood Cliffs, NJ, USA, 1977.

[2] B. Bükcü and M. K. Karacan, "On the involute and evolute curves of the spacelike curve with a spacelike binormal in Minkowski 3-space," International Journal of Contemporary Mathematical Sciences, vol. 2, no. 5-8, pp. 221-232, 2007.

[3] B. Bükcü and M. K. Karacan, "On involute and evolute curves of spacelike curve with a spacelike principal normal in Minkowski 3-space," International Journal of Mathematical Combinatorics, vol. 1, pp. 27-37, 2009.

[4] B. Bükcü and M. K. Karacan, "On the involute and evolute curves of the timelike curve in Minkowski 3-space," Demonstratio Mathematica, vol. 40, no. 3, pp. 721-732, 2007.

[5] G. S. Birman and K. Nomizu, "Trigonometry in Lorentzian geometry," The American Mathematical Monthly, vol. 91, no. 9, pp. 543-549, 1984.

[6] B. O’Neill, Semi-Riemannian Differential Geometry, Academic Press, New York, NY, USA, 1983. 


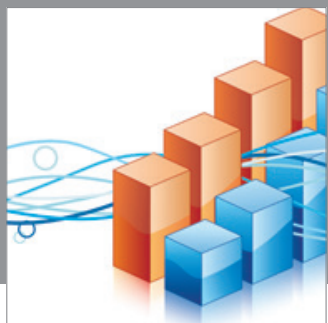

Advances in

Operations Research

mansans

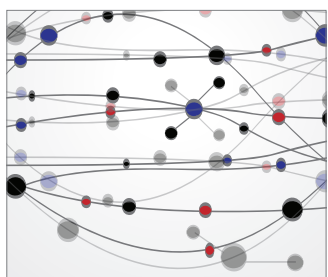

The Scientific World Journal
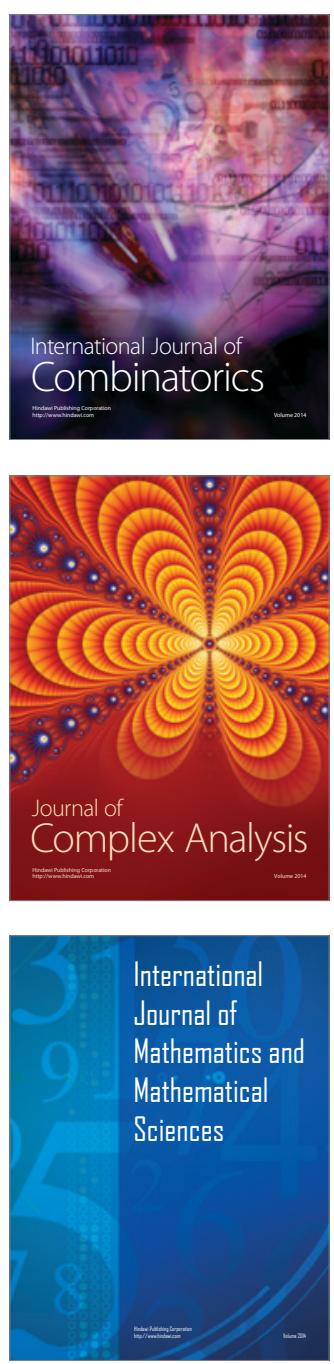
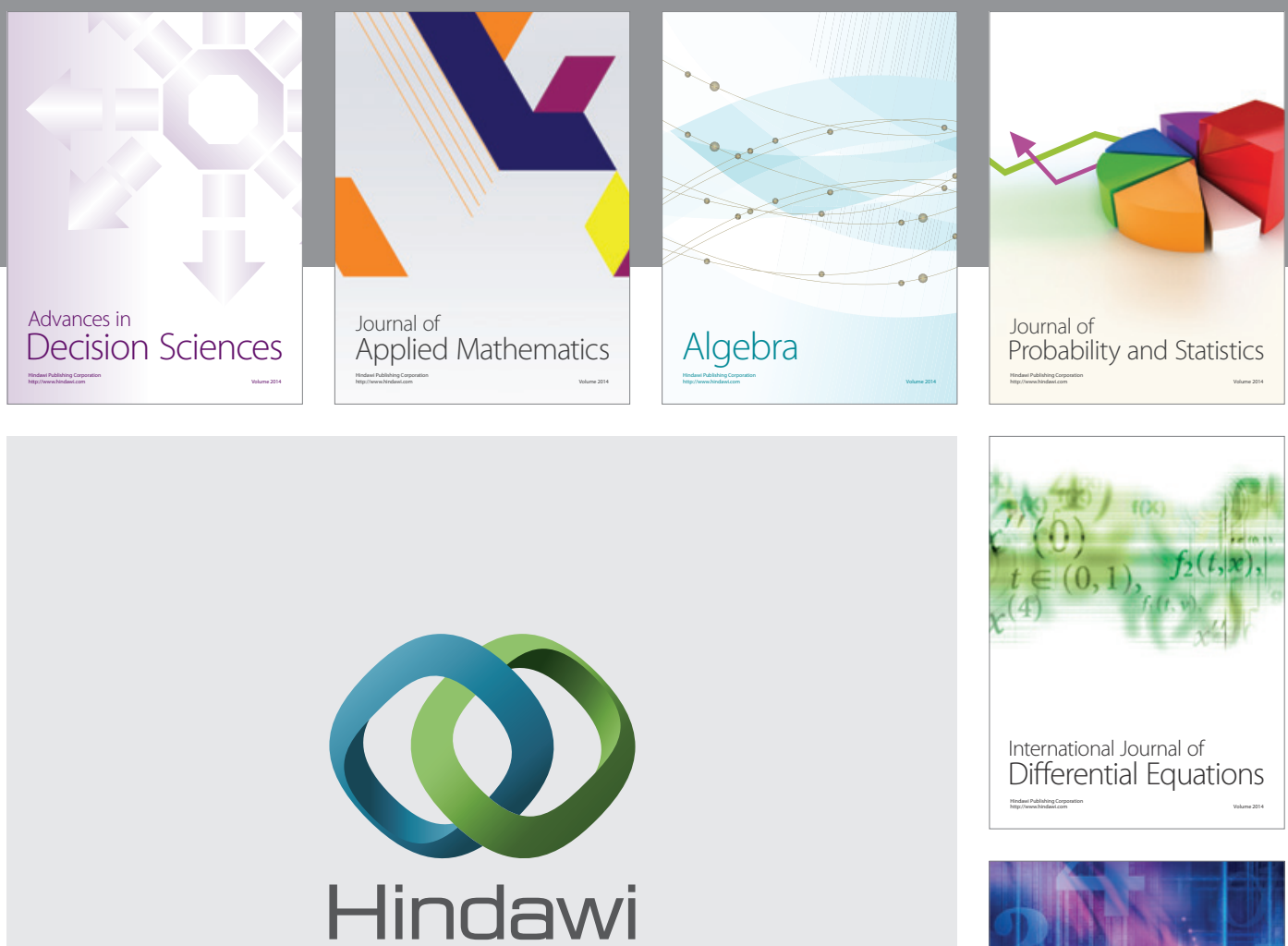

Submit your manuscripts at http://www.hindawi.com
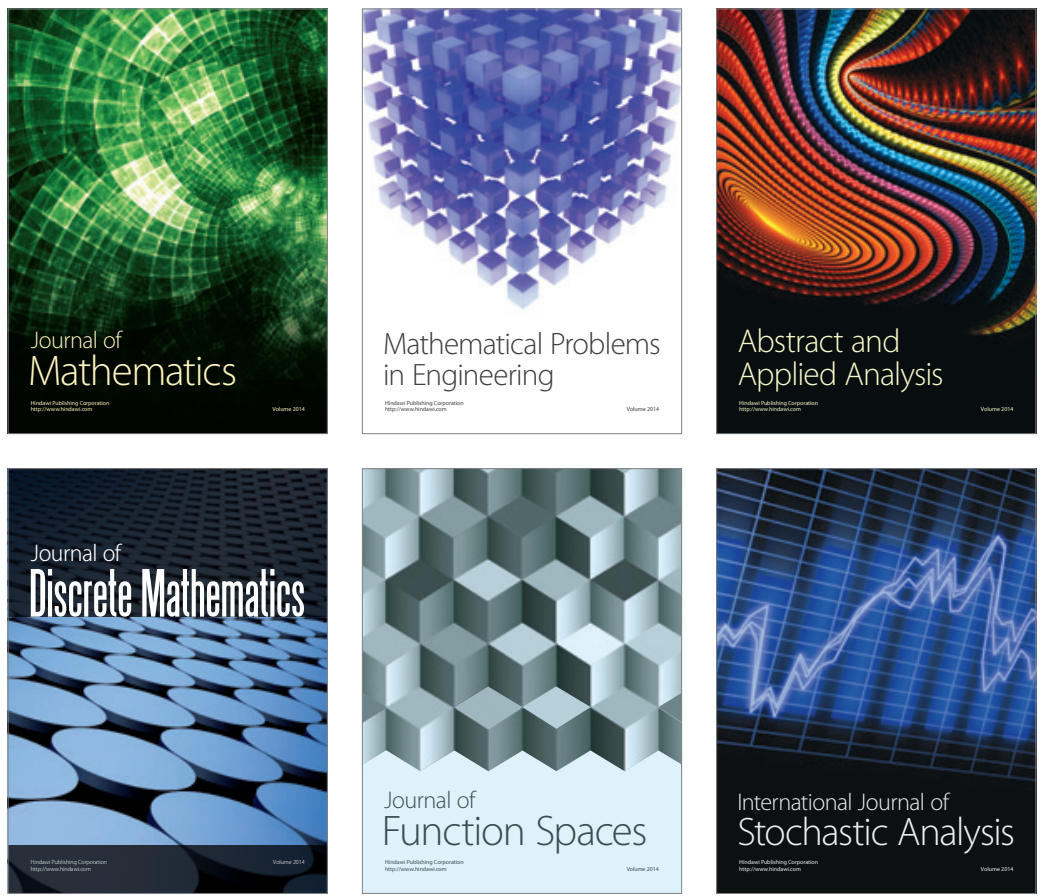

Journal of

Function Spaces

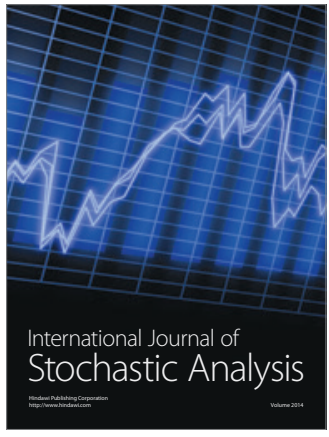

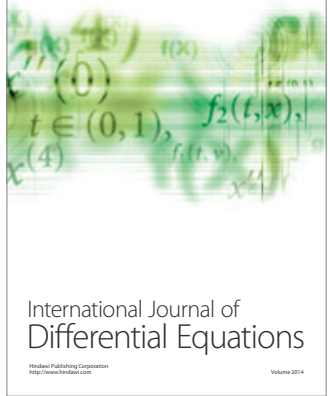
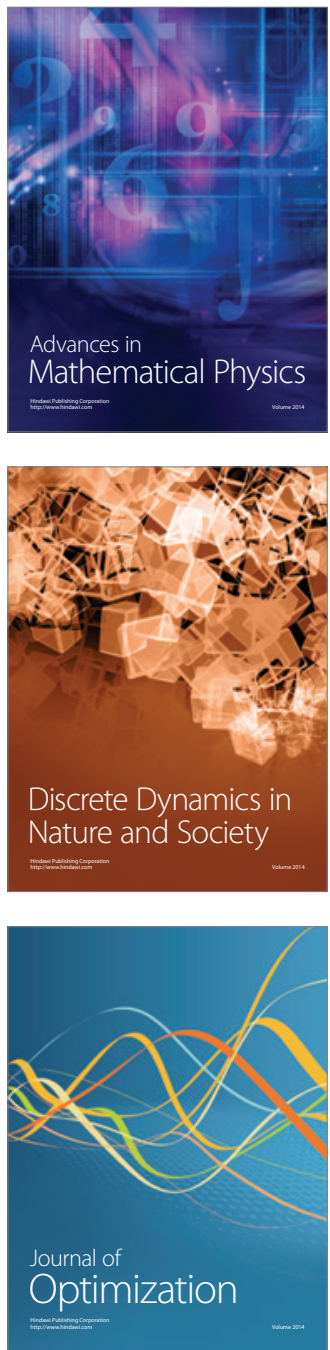